\title{
RESEARCH
}

Open Access

\section{Indications and complications of inpatient parenteral nutrition prescribed to children in a large tertiary referral hospital}

\author{
C. Mantegazza ${ }^{1,2^{*}}$ (D, N. Landy ${ }^{1}$, G. V. Zuccotti ${ }^{2}$ and J. Köglmeier ${ }^{1}$
}

\begin{abstract}
Background: Parenteral Nutrition (PN) is prescribed to children with intestinal failure. Although life saving, complications are common. Recommendations for indications and constituents of PN are made in the 2005 guidelines by the European Society of Paediatric Gastroenterology, Hepatology and Nutrition (ESPGHAN). The aim of this study was to establish if the indications for prescribing PN in a tertiary children's hospital were appropriate, and to identify complications encountered. Data were compared to those published by the National Confidential Enquiry into patient outcome and death (NCEPOD) carried out in the United Kingdom in 2010.
\end{abstract}

Methods: Children and newborns receiving inpatient PN over a 6 months period were entered into the study and data was collected prospectively. The appropriate indications for the use of PN were based on the ESPGHAN guidelines. Recorded complications were divided into metabolic, central venous catheter (CVC) related, hepatobiliary and nutritional.

Results: A total of 303 children (67 newborns) were entered into the study. The main indications for the start of PN were critical illness (66/303), surgery (63/303) and bone marrow transplantation (28/303). The ESPGHAN recommendations were followed in $91.7 \%(278 / 303)$ of cases ( $95.5 \%$ of newborns, $90.7 \%$ of children). PN was considered inappropriate in 12/303 patients and equivocal in 13. The mean PN duration was 18 days (1-160) and the incidence of complications correlated to the length of PN prescribed. Metabolic, hepatobiliary and CVC related complications affected $74.6,24.4,16.4 \%$ of newborns and $76.7,37.7$ and $24.6 \%$ of children respectively. In relation to the appropriate indications for the start of PN our results mirrored those reported by the NCEPOD audit (92.4\% of newborns and $88.6 \%$ children). However, the incidence of metabolic disturbances was higher in our cohort ( $74.6 \%$ vs $30.4 \%$ in children, $76.7 \%$ vs $14.3 \%$ in newborns) but CVC related complications lower amongst our newborns (16,4\% vs $25 \%)$.

Conclusions: Although the indications for inpatient PN in children is mostly justified, there is still a proportion who is receiving PN unnecessarily. PN related complications remain common. There is a need for better education amongst health professionals prescribing PN and access to nutritional support teams to reduce unwanted side effects.

Keywords: Pediatric parenteral nutrition, ESPGHAN guidelines, indications, appropriatness, complications, NCEPOD

\footnotetext{
*Correspondence: mantegazza.cecilia@asst-fbf-sacco.it;

cecimante@hotmail.com

${ }^{1}$ Great Ormond Street Hospital for Children NHS Foundation Trust, London,

UK

${ }^{2}$ Department of Pediatrics, University of Milan, Ospedale dei Bambini Vittore

Buzzi, Milan, Italy
}

(c) The Author(s). 2018 Open Access This article is distributed under the terms of the Creative Commons Attribution 4.0 International License (http://creativecommons.org/licenses/by/4.0/), which permits unrestricted use, distribution, and reproduction in any medium, provided you give appropriate credit to the original author(s) and the source, provide a link to the Creative Commons license, and indicate if changes were made. The Creative Commons Public Domain Dedication waiver (http://creativecommons.org/publicdomain/zero/1.0/) applies to the data made available in this article, unless otherwise stated. 


\section{Background}

Parenteral Nutrition (PN) is used when nutrition cannot be tolerated via the oral or enteral route [1]. Since its first use in the late 1960s PN has become a well established therapy and a lifesaver in well selected hospitalised newborns and children with intestinal failure (IF) or those who fail to meet their nutritional requirements with enteral intake alone [2].

However, PN is bay far no panacea, quite invasive and associated with potentially fatal complications [3]. Central venous catheter (CVC) related infection, occlusion, central venous thrombosis, pulmonary embolism and accidental catheter removal or damage, electrolyte disturbances and renal abnormalities, metabolic bone disease, micronutrient deficiencies and liver disease are well known complications of $\mathrm{PN}[3,4]$.

It is hence crucial to weigh the potential benefits against the risks to the patient, led alone the cost implications to the health care system [5].

In 2005 the European Society of Paediatric Gastroenterology, Hepatology and Nutrition (ESPGHAN) and the European Society for Clinical Nutrition and Metabolism, supported by the European Society of Paediatric Research, have published guidelines on the use of PN in children, aiming at identifying the most common and reasonable indications for the use of $\mathrm{PN}$ in newborns, infants and children in order to reduce its inappropriate use [6]. The authors make recommendations when to start PN and how to minimise the related complications [6].

Much is published on the use and incidence of complications occuring in adults receiving intravenous nutrition in hospital [7, 8]; however little is known in children [6].

In 2010 the National confidential enquiry into patient outcome and death (NCEPOD) conducted a retrospective survey on a random sample of patients receiving $\mathrm{PN}$ in hospital in the United Kingdom including children and newborns [9]. The purpose of this enquiry was to reveal areas of deficiency in the use of $\mathrm{PN}$ in hospitalised patients and to make recommendations how to resolve them [9]. The neonatal cohort included 264 subjects but the audit in the paediatric age group consisted of just 70 patients [9]. In the neonatal cohort $92,4 \%$ of the indications for PN were considered appropriate [9]. In the paediatric age group (1 month to 19 years) $88 \%$ of children were given PN for justified reasons [9]. Moreover the audit showed that in children complications associated with PN still frequently occur: metabolic disturbances affecting $30,4 \%$ of children and 14,3\% of newborns, and CVC related complications encountered in $25 \%$ of newborns, were amongst the most common ones [9]. The need of a large scale audit of PN care in children was hence suggested [9].

The aim of this observational study was hence to get a better understanding why children in hospital are started on PN and if the indications, based on the 2005 ESPGHAN guidelines, are appropriate and to estimate the incidence of $\mathrm{PN}$ related complications. We wanted to compare our results to the NCEPOD audit and, if needed, develop strategies to avoid inappropriate PN prescribing in the future.

\section{Methods}

All newborns, infants and children admitted to Great Ormond Street Hospital who started PN were identified from the pharmacy database and enrolled prospectively over a 6 months study period between October 2013 and March 2014. Patients established on home PN were excluded. Information was obtained from the PN prescription, medical, nursing and dietetic records.

\section{Indications for starting PN}

Patient demographics, weight and height/length, referring department (medical, surgical, intensive care), underlying diagnosis, current pathology, feeding regime prior to and on PN, reason for starting PN and duration were recorded. Under-nutrition in children was defined according to the World Health Organistation criteria [10]. The indications for prescribing PN were divided according to specialty into medical and surgical, and primary or secondary IF. Primary IF was defined as requirement for intravenous nutrition due to an underlying anatomical, structural gut pathology; IF was considered secondary if PN was started as a consequence of other conditions leading to enteral feed intolerance. The indications for starting PN were classified as appropriate, inappropriate or indeterminate based on the ESPGHAN 2005 guidelines [6]. In newborns with a very low or extremely low birth weight indications for PN were based on separate guidelines applicabable to this specific group of patients [11] (Tables 1 and 2).

\section{Table 1 Appropriate indications for PN in infants and children [6] \\ $1 \quad$ Infants aged 1 to 12 months: inability to achieve an adequate energy intake ${ }^{a}$ for more than 3 days \\ 2 In children older than 1 year: inability to achieve an adequate energy intake ${ }^{a}$ for more than 5 days \\ 3 Children not expected to meet an appropriate energy and nutrient intake for more than 7 days \\ 4 Clinical condition leading to an absolute or relative contraindication to enteral nutrition ${ }^{b}$ \\ $5 \quad$ In children older than 1 year: duration of PN of at least 5 days except in undernourished children}

adeguate energy intake defined as $60-80 \%$ of the Kcal for age [5]

babsolute contraindications to enteral nutrition: paralytic or mechanical ileus, anatomical disruption of the gastrointestinal (Gl) tract, intestinal obstruction, necrotising enterocolitis, GI ischemia, diffuse peritonitis, bowel perforation or a state of severe shock $[5,16,90]$

Relative contraindications to enteral nutrition: gastro-intestinal bleeding, intractable diarrhea, enteric fistula, toxic megacolon, gastro-intestinal dysmotility, severe vomiting $[5,16,90]$ 
Table 2 Appropriate indications for PN in newborns [5, 11]

\begin{tabular}{ll}
\hline 1 & $<32$ weeks of gestation and/or $<1500 \mathrm{~g}$ \\
2 & $>32$ weeks of gestation with IF \\
3 & Bridging PN \\
\hline
\end{tabular}

The use of $\mathrm{PN}$ in relation to appropriateness was considered indeterminate if a judgement could not be made due to lack of enough clinical information to be able to reach a conclusion if $\mathrm{PN}$ was used appropriately, if EN was achievable but not used because of concerns over potential complications associated with feeding or a delay in passing a suitable enteral feeding tube, eg if the child did not cooperate with the insertion of a nasal feeding device or if jejunal feeding was required but jejunal access was unsuccesful.

\section{PN Complications}

Bloods for vitamins (A,D,E), folate, trace elements (zinc, selenium, copper, iron and mangnesium) and ferritin were sent when patients were started on PN.

Electrolites, urea, peripheral blood glucose, carbon dioxide $(\mathrm{CO} 2)$ and $\mathrm{pH}$ were monitored daily for at least 10 days; subsequent testing was based on the patients clinical needs. Liver function tests (LFTs) including alanine aminotransferase (ALT), aspartate aminotransferase (AST), alkaline phosphatase (ALP), gamma-glutamyl transpeptidase (GGT) and bilirubin, urinary sodium (uNa) and triglycerids (TG) were performed at least weekly. In children who remained on $\mathrm{PN}$ for more than 28 days vitamins and trace elements were checked after one month of nutritional support and continued to be monitored every four weeks for the duration of PN.

All children were followed on a daily basis and any complications recorded.

Complications were divided into metabolic, CVC related, nutritional and hepatobiliary and defined as follows:

1) metabolic: deficiency or excess of electrolytes, hypo and hyperglycemia, hypercapnia, acid-base disturbances, rise in urea, hypertriglyceridemia and low uNa;

- electrolytes abnormalities were defined as at least two consecutive results above or below the reference value for age and divergent from baseline levels, requiring a change of PN prescription or oral/iv supplementation; calcium and magnesium were corrected for the albumin level, serum sodium was corrected in the presence of hyperglycemia [12]. Patients were monitored for hypophospathemia throughout the duration of PN, which was considered a marker of refeeding syndrome (RS) in the first 10 days of $\mathrm{PN}$ in malnourished children or those who had lost a significant amount of weight prior to the start of PN [13].

- Hypoglycaemia was defined as a blood glucose level of less than $3.6 \mathrm{mmol} / \mathrm{l}(40 \mathrm{mg} / \mathrm{dl})$ both in newborns and in children [14, 15]. Children were considered hyperglycaemic if their blood sugare rose above $7 \mathrm{mmol} / \mathrm{L}$ (126 mg/dl) [14]. In newborns plasma glucose concentrations above $8 \mathrm{mmol} / \mathrm{l}(150 \mathrm{mg} / \mathrm{dL})$ were considered pathological in accordance with the guidelines of the American Society for Parenteral and Enteral Nutrition [14].

- Blood triglyceride concentrations were checked on a weekly basis $4 \mathrm{~h}$ after stopping the lipid infusion; levels $>200 \mathrm{mg} / \mathrm{dl}$ in newborns, > $250 \mathrm{mg} / \mathrm{dl}$ in infants and $>400 \mathrm{mg} / \mathrm{dl}$ in children were considered pathological [16].

- uNa was considered low if $<20 \mathrm{Meq} / \mathrm{L}$.

- A rise in urea due to renal failure or gastrointestinal bleeding was not recorded [17, 18].

- Acid-base disturbances as a consequence of respiratory failure, sepsis and metabolic disease were not considered to be related to PN.

- Hypercapnia secondary to respiratory failure was not recorded.

2) CVC related complication: Exit site and central line associated bloodstream infections (CLABSIs), line colonisation, occlusion, thrombosis, damage, misplacement, phlebitis, extravasation and venous thrombosis; CLABSIs were defined according to the 2012 guidelines of Centers for Disease Control and Prevention [19]. All organisms causing CLABSIs were recorded.

3) Nutritional complications: deficiency or excess of vitamins and trace elements after 30 days of PN and divergent from baseline values were recorded; Vitamin concentrations were measured by high pressure liquid cromatography (HPLC). Ferritin was used as a marker of iron storage in the presence of a normal C-reactive protein [20]. As ferritin syntesis is enhanced by cytokines, high values were not considered due to iron overload when inflammation was present or the inflammatory markers were elevated [20].

In particular, if data were available, we followed ESPGHAN guidelines for reference values:

- we defined a high vitamin $E$ level as greater than $3.5 \mathrm{mg} / \mathrm{dl}$ and low Vitamin E levels $<1 \mathrm{mg} / \mathrm{dL}$ and $<$ $0.5 \mathrm{mg} / \mathrm{dl}$ in premature infants and children respectively [6,21, 22].

- 300-800 microG/L were considered normal reference values for vitamin $\mathrm{A}$ in children older than 
6 months of age. Serum concentrations below 200 microg/L were considered to indicate deficiency in premature infants [6].

We used reference values obtained from the ESPGHAN guidelines considered normal for sex and age if no data was available from our laboratory.

4) Hepatobiliary complications: any prolonged (at least two weeks) rise in LFTs not explained by other causes (eg medication, metabolic disorder, haemolysis, surgical obstruction) $1.5 \times$ above the normal value for age was recorded. Hyperbilirubinaemia was defined as $>50 \mathrm{micromol} / \mathrm{L}$.

The study was approved by the Ethical Committee of Great Ormond Street Hospital and informed consent was obtained from parents or legal guardians of the patients. All data collected were analysed using Microsoft Access and Excel.

\section{Results}

\section{Population characteristics}

Over a 6 months period PN was prescribed to 318 newborns and children; fifteen patients were excluded as already established on home PN. A total of 303 were hence included in the study; 236 (77.9\%) were older than 1 month of age, $67(22,1 \%)$ were newborns; the median age was 38 months (range 0-223), 54.5\% were females, 89 patients were malnourished $(29,4 \%)$.

Only 17 patients did not have a pre-existing diagnosis, 90 children suffered from congenital heart disease, 68 had a diagnosis of malignancy $(54,4 \%$ leukemia, $46,6 \%$ solid tumour) and 41 had a known pathology of the GI tract. All the underlying diagnoses are listed in Table 3.

Table 3 Underlying diagnosis

\begin{tabular}{lll}
\hline Pre-existing diagnosis & No & Percentage \\
\hline Cardiac disease & 90 & 29,7 \\
Malignancy & 68 & 22,4 \\
Gl pathology & 41 & 13,5 \\
Immune dysfunction & 20 & 6,6 \\
No pre existing pathology & 17 & 5,6 \\
Metabolic disorder & 16 & 5,3 \\
Prematurity & 16 & 5,3 \\
Neurological abnormality & 15 & 5,0 \\
Syndrome & 7 & 2,3 \\
Endocrine disorder & 3 & 1,0 \\
Congenital bone diseases & 3 & 1,0 \\
Lung disease & 3 & 1,0 \\
Rheumatological disorder & 3 & 1,0 \\
\hline
\end{tabular}

Children were referred for PN from medical (34\%, majority oncology $57 / 103)$, surgical (35\%, majority cardiothoracic 81/106) and Intensive Care Units (31\%). Main indications for prescribing PN were critical illness (66/ $303)$, pre, peri and post surgery nutritional support (63/ $303)$ and bone marrow transplantation (28/303).

The majority of children (57.4\%) who were prescribed PN had secondary IF as a consequence of an acute or chronic medical condition. Multi-organ, respiratory, renal or heart failure, shock, sepsis and macrophage activation syndrome were the most common causes $(21.8 \%)$ followed by bone marrow transplantation $(9.8 \%)$ or feed intolerance due to chemotherapy $(8.2 \%)$ or radiation induced mucositis. The indications for PN are listed in Table 4.

At the end of the study all but 15 patients were weaned off PN. The mean duration of PN was 18 days (range from 1-160 days), 55 patients $(18,2 \%)$ had more than 28 days of intravenous support.

\section{Appropriatness of indications for prescribing PN}

PN was considered appropriate in 278/303 (91,7\%), inappropriate in $12(4,0 \%)$ and indeterminate in $13(4,3 \%)$ patients.

PN was defined inappropriate in 5 non malnourished children older than 12 months who had less than 5 days of PN where it was not anticipated at the start that PN

Table 4 Indications for PN

\begin{tabular}{lll}
\hline Indication & No & Percentage \\
\hline Critical illness & 66 & 21.8 \\
Pre-peri-post surgery nutritional support & 63 & 20.8 \\
Bone marrow transplantation & 28 & 9.2 \\
Feed intolerance & 25 & 8.2 \\
NEC & 22 & 7.3 \\
Intestinal resection in congenital malformation of the & 21 & 6.9 \\
Gl tract & & \\
Mucositis due to chemotheraphy & 19 & 6.3 \\
Other intestinal resections & 10 & 3.3 \\
Condition associated with malabsorption & 9 & 3.0 \\
Decompensation in patients with metabolic condition & 7 & 2.3 \\
Organ transplant & 6 & 2.0 \\
Post surgical ileus & 4 & 1.3 \\
Autoimmune disorder associated with intestinal failure & 4 & 1.3 \\
Gl dysmotility & 4 & 1.3 \\
Miscellaneus & 4 & 1.3 \\
Post operative nutritional support & 3 & 1.0 \\
Malnourishment & 3 & 1.0 \\
Meconium aspiration & 2 & 0.7 \\
Acute pancreatitis & 2 & 0.7 \\
Heart cachexia & 1 & 0.3 \\
\hline & & \\
\hline
\end{tabular}


would be required for longer; moreover four infants were given PN despite a functional GI tract and one was kept nil by mouth and given PN for one day for query NEC but started again on feeds the following day. Finally two term newborns were precribed PN unnecessarily as they were able to tolerate $>80 \%$ of their requirements enterally when PN was started and PN was only given for one day.

According to age PN was defined as appropriate in $95,5 \%$ of newborns and in $90,7 \%$ of infants and children.

Our results were similar to the data reported in the NCEPOD audit [9] Table 5.

\section{Complications}

A total of 239/303 $(78,9 \%)$ patients developed complications as a consequence of $\mathrm{PN}$; the mean duration of $\mathrm{PN}$ in these children was 22 days (range 2-160) whereas those who experienced no complications received PN for a mean of 5 days (range 1-23). All patients who had PN for more than 28 days developed complications.

Metabolic complications were the commonest occuring in $76.2 \%$ of patients: hyperphosphatemia was the most frequent (111 patient, 36.6\%), followed by hypermagnesemia $(31,4 \%)$ and hypercalcemia $(29,7 \%)$. In particular hyperphosphatemia developed in $8 / 89$ malnourished patients. Hypophosphatemia was seen in 84 patients $(27,7 \%)$ and $66 / 84(59,9 \%)$ developed it in the first 10 days of PN administration. RS was blamed in $13 / 66$ patients as they were malnourished or had lost a significant amount of weight prior to the start of PN. However, most malnourished patients $(85.4 \%)$ did not develop hypophosphatemia in the first days after being started on intravenous support.

Hyperglycemia and hypoglycemia affected 32 (10.6\%), and $9(3.0 \%)$ of all children respectively. Twenty-seven (8.9\%) children developed hypercapnia. Acid-base disturbances were noted in 55 children, 36/303 (11.9\%) developed acidosis.

All metabolic complications are listed in Table 6.

Hepatobiliary complications were found in 106 patients (35,0\%). A rise in ALT was the commonest affecting 82/303 (27.1\%). Elevation of GGT occured in 38 children (12,5\%); an abnormal bilirubin in 31 (10,5\%). Rise in ALP was documented in 14 patients. Just 5 had an elevated AST. Twenty-one patients with a derangement of LFTs had a
Table 6 Metabolic complications

\begin{tabular}{lll}
\hline Metabolic complication & No & $\begin{array}{l}\text { Percentage among } \\
\text { all patients }\end{array}$ \\
\hline Hyperphophatemia & 111 & 36,6 \\
Hypermagnesemia & 95 & 31,4 \\
Hypercalcemia & 90 & 29,7 \\
Hypophosphatemia & 84 & 27,7 \\
Rise in urea & 74 & 24,4 \\
Hypokalemia & 70 & 23,1 \\
Hypophosphatemia in the first 10 days & 66 & 21,8 \\
Hypernatremia & 36 & 11,9 \\
Acidosis & 36 & 11,9 \\
Hyponatremia & 35 & 11,6 \\
Hyperkalemia & 32 & 10,6 \\
Hyperglycemia & 32 & 10,6 \\
Hypercarboxinemia & 27 & 8,9 \\
Hypomagnesemia & 25 & 8,3 \\
Hypertrigliceridemia & 21 & 6,9 \\
Hypocalcemia & 19 & 6,3 \\
Alcalosis & 19 & 6,3 \\
Hypercloremia & 14 & 4,6 \\
Hypocloremia & 13 & 4,3 \\
Low urinary sodium & 12 & 4,0 \\
Hypoglycemia & 9 & 3,0 \\
\hline & & \\
\hline & 62,6 \\
\hline
\end{tabular}

concomitant line infection, $45 / 106(42,5 \%, 14.8 \%$ of the total population) had a rise in more than one liver parameter. The mean duration of PN in patients who developed hepatobiliary complications was 31 days versus 12 days in children who did not experience any alteration in LFTs.

CVC related complications affected 69 (22,8\%) children, the commonest being blockage of the line (36, $11.6 \%)$. Thirty-one children (10.2\%) developed a line infection (no child had more then one episode). The principle organism involved was coagulase negative staphylococcus grown in 17/31 (54,8\%) blood cultures followed by Streptoccocus species $(5 / 16,1 \%)$ and Candida species (3, 9.7\%). Line infections with gram negative organisms occured in 4 children (12.9\%) and was caused by Enterobacteriacae (2/4), Escherichia coli (1/4) and Pseudomonas Aeruginosa (1/4). The mean duration of

Table 5 Appropriatness of PN divided by age and compared to the NCEPOD audit

\begin{tabular}{|c|c|c|c|c|}
\hline & \multicolumn{2}{|l|}{ Current Audit } & \multicolumn{2}{|l|}{ NCEPOD } \\
\hline & Newborns & Infants/children & Newborns & Infants/children \\
\hline & No, Percentage & No, Percentage & No, Percentage & No, Percentage \\
\hline Appropriate & $64,95.5$ & $214,90.7$ & $244,92.4$ & $62,88.6$ \\
\hline Indeterminate & $1,1.5$ & $12,5,1$ & $4,1.5$ & / \\
\hline Inappropriate & $2,3.0$ & $10,4.2$ & $16,6.1$ & $8,11.4$ \\
\hline
\end{tabular}


PN in children who developed a line infection was 37 days versus 17 days in children who did not develop CLABSIs. The central venous catheter was subsequently removed and replaced with a new line in 11/31(15.9\%) patients. Colonisation of the line was found in $9 / 31$ patients, mostly with Coagulase negative Staphylococcus $(5 / 9,55.6 \%)$. Four children experienced a catheter breakage which required repair.

Thirty-five/303 (11,6\%) children developed nutritional complications. Of the 55 children who received $\mathrm{PN}>28$ days, 37 had nutritional bloods performed. 35/37 (94,6\%) showed a deficiency or excess in the levels of fat soluble vitamins and/or trace elements. The most common abnormal results seen were a rise in vitamin $\mathrm{A}$ and $\mathrm{E}$ levels and low levels of selenium with $27.3 \%, 25.5 \%$ and $29.1 \%$ of patients affected respectively. All the nutritional complications encountered are listed in Table 7.

According to age metabolic complications affected $74.6 \%$ of all newborns, the commonest being hyperphophatemia (33/67) and hypermagnesemia (30/67), and $76.7 \%$ of all children. Hyperglycaemia developed in $10.45 \%$ of newborns, and hypoglycemia in $6.0 \%$. Seventeen $(25.4 \%)$ of newborns and $89(37.7 \%)$ of children showed a derangement in their LFTs.

Eleven newborns $(16.4 \%)$ and 58 children $(24.6 \%)$ developed CVC related complications, in particular 4 newborns had a culture positive CLABSIs.

Nutritional complications developed in 3 newborns and 32 children.

Table 7 Nutritional complications

\begin{tabular}{lll}
\hline Nutritional complication & No & $\begin{array}{l}\text { Percentage among all patients } \\
\text { with PN }>28 \text { days }\end{array}$ \\
\hline Vitamins & 15 & 27,3 \\
High vitamin E & 14 & 25,5 \\
High vitamin A & 1 & 1,8 \\
High vitamin D & 1 & 1,8 \\
Low vitamin D & 7 & 12,7 \\
High folate & 1 & 1,8 \\
High vitamin B12 & & \\
Trace elements & 7 & 12,7 \\
Low Zinc & 1 & 1,8 \\
High Zinc & 16 & 29,1 \\
Low selenium & 2 & 3,6 \\
High selenium & 9 & 16,4 \\
Low copper & 5 & 9,1 \\
High copper & 4 & 7,3 \\
Low iron & 1 & 1,8 \\
High iron & 1 & 1,8 \\
High Manganese & & \\
\hline
\end{tabular}

Comparison between our audit and the NCEPOD is reported in Table 8 [9].

\section{Discussion}

Feeding children with a non functioning gut intravenously or those who are unable to meet their nutritional requirements enterally is a well established practice. However, PN is by far no panacea and its use may cause serious complications to the patient and put a huge financial burden to the health care system [3, 4, 23], The 2005 ESPGHAN guidelines make recommendations when PN should be prescribed to children [6]. In 2010 the NCEPOD conducted a national retrospective audit in the UK on adults and children receiving PN: indications for PN were considered appropriate in $92.4 \%$ of newborns and in $88.6 \%$ of children aged $>1$ month [9]. Complications were demonstrated to be common. Metabolic disturbances occured in 30,4\% of children and $14,3 \%$ of newborns of which $25 \%$ also developed CVC related complications [9].

The NCEPOD audit revealed suboptimal care in the administration of PN in newborns and children and pointed out the importance of the Nutrition Support Team (NST) [9]. However, as only 70 children were included in the data analysis, a larger scale audit of PN care in children was recommended [9].

The findings of our study mirror those of the NCEPOD; yet our data was collected prospectively and based on a much bigger cohort of 236 children. Although our neonatal group was smaller (67 versus 264 NCEPOD) we had similar results in this age group as well. In our cohort the use of PN was appropriate in $95.5 \%$ of newborns and $90.5 \%$ of children (aged 1 month-18 years). We observed a high complication rate with $78.9 \%$ of patients being affected. Similar to the NCEPOD audit, metabolic disturbances were the most frequently occuring problem and exceeded the percentage of children affected in the 2010 retrospective NCEPOD study. However, CVC related complications were less common in our population compared to the NCEPOD audit. Amongst our patients 16.4\% experienced problems with their catheter compared to $25.0 \%$ in the NCEPOD group. However, complications were poorly defined in the NCEPOD study and comparison between the two studies is hence difficult. Of note is the fact that PN continues to be prescribed inappropiately in a percentage of children which may lead to serious or even life-threatening complications. All effort should hence be made to avoid the use of PN if clinically not clearly indicated.

\section{Inappropriate prescribing of PN}

In our cohort PN was inappropriately prescribed to children with a functional gut when enteral feeds were not tried vigorously enough and in situations associated with 
Table 8 Comparison between our audit and NCEPOD

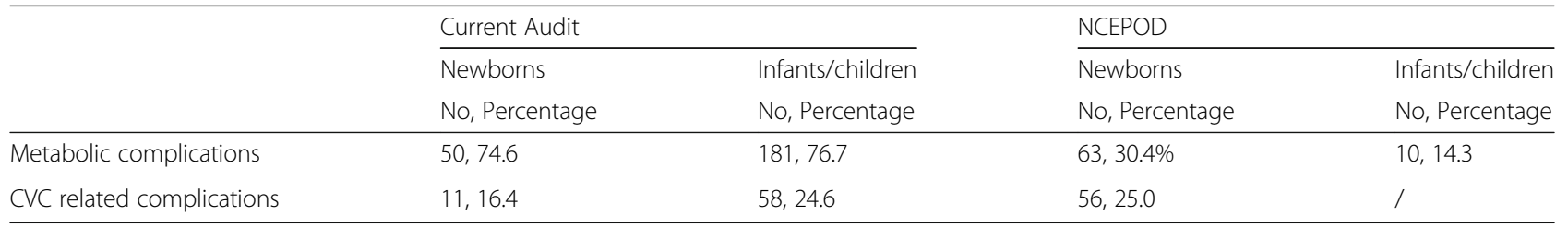

a temporary feed intolerance. In particular in children older than 12 months of age, with a good nutritional status, post operative $\mathrm{PN}$ is considered beneficial only if the child is expected to remain nil by mouth for more than 7 days and when PN is administered for at least 5 days, otherwise the risk associated with the administration outweighs the benefits [9, 24-26].

\section{Main Metabolic complications: hyperphosphatemia, hypercalcemia, hypermagnesemia, hypophosphatemia} The commonest metabolic complications seen in our patients were an elevated phosphate, magnesium and calcium. These minerals have a crucial role in the cellular physiology of the neuromuscolar and cardiovascular systems [27]. Derangement can lead to significant pathologies including respiratory failure, disruption of the cardiac conduction system with arrhythmias, complete heart block, and in severe cases cardiac arrest and coma [27-35]. In the oncology children rumour lysis syndrome may have contributed to these abnormal results [29]. An overawareness of RS could have also led to overprescribing of electrolytes. Given that only a small number of malnourished patients (9.0\%) developed hyperphosphatemia this appears unlikely though. Hypophosphatemia was also commmonly observed in our population with $23.1 \%$ of children/newborns affected. Hypophosphatemia is associated with respiratory insufficiency and decreased myocardial contractility, rhabdomyolysis, hemolysis, impaired platelet and white blood cell function, and neurological abnormalities including seizures [36, 37]. Hypophosphatemia during the first 7-10 days on PN may also be a consequence of RS, a potentially lethal condition which occurs in starved, malnourished or ill metabolically stressed patients when too much nutrition is provided initially [13, 38]. Based on this definition we found that up to $21.8 \%$ of our patients were affected but amongst them just $19.7 \%$ were malnourished (4,3\% of the total population). A study from 2016 on RS in adults receiving artificial support reported only a $2 \%$ incidence. However RS was more rigorously defined not only by the finding of hypophasphatemia [39]. Data collected retrospectively on children in the same year showed that hypophosphatemia, hypokalemia and hypomagnesimia were frequent in children receiving inpatient PN before and during the first four days after starting PN [40]. Malnourished children in this cohort were found to be more prone to develop low levels of potassium, wheras patients who received a high intravenous protein load had a higher incidence of hypomagnesaemia [40].

More than $15 \%$ of newborns who were entered into the NCEPOD audit experienced low phosphate levels [9]. However, the authors do not comment whether this was felt to be a consequence of RS or not. In our cohort a large proportion of malnourished patients (85.4\%) did not develop hypophosphatemia in the first days after starting PN. It is standard practice in our hospital to give extra phosphate at the start of $\mathrm{PN}$ in children who are at high risk of developing RS. The calorie intake is built up gradually over a few days whilst children are carefully monitored which may explain the lower incidicence of hypophosphataemia in our patients.

\section{Hyperglycemia}

An excess in serum glucose levels affected $10.6 \%$ of our patients. Hyperglycaemia is associated with a higher infection rate [41], can lead to overfeeding [42, 43] and even death [44-46]. A glucose intake exceeding the maximal oxidation rate promotes fat synthesis and deposition $[47,48]$ with a large increase in CO2 production [49]. Hypercapnia, which was found in $8.9 \%$ of our patients, is compensated by increasing the respiratory rate or depth of breathing, which is well tolerated by stable patients but may lead to complications in patients with respiratory compromise [49]. In addition the overfeeding of glucose can have a negative impact on liver function, lead to hepatic steatosis and a rise in serum transaminases [50,51]. Neonates receiving PN are particularly vulnerable to the consequences of hyperglycemia which is positively correlated to high morbidity and mortality, especially in premature and low birth weight infants $[52,53]$. As this age group is prone to developing low blood sugar levels large amounts of glucose are often administered to neonates receiving PN which can easily cause hyperglycemia if children are not monitored closely $[52,53]$. Unlike the NCEPOD audit, which showed a $>20 \%$ prevalence of hyperglycemia in newborns, we only saw elevated glucose levels in $10.45 \%$ of our newborns. Although we were encouraged by these results there is still a significant amount of work required to improve blood sugar control in these most vulnerable children. The guidelines of the American Society 
for Parenteral and Enteral Nutrition suggest that newborns should receive intravenous lipids when dextrose is infused, as glycerol is the predominant gluconeogenic substrate $[54,55]$ and to consider insulin in cases of prolonged hyperglycemia [56].

\section{Hepatobiliary complications}

In pediatric patients receiving $\mathrm{PN}$, various definitions of PN-associated cholestasis (PNAC) and intestinal failureassociated liver disease (IFALD) are used in the literature [57]. There is hence a great variation in the prevalence of hepatic complications reported ranging from $7.4 \%$ to $84 \%$ in children [58-60]. In infants the prevalence of PNAC appears higher with $30 \%-70 \%$ being affected [58, 61, 62]. In keeping with the definition of the Hepatology working group of the Bristish Society of Paediatric Gastroenterology, Hepatology and Nutrition we considered IFALD as the elevation of LFTs including AST, ALT, GGT, ALP $\times 1.5$ above the reference range for age and a bilirubin level $>50$ micromoli/L for [61]. The BPSGHAN position paper consideres persistently elevated LFTs for a minimum of 6 weeks abnormal [61. However a ESPGHAN position paper from 2015 defines early IFALD as the presence of hyperbilirubinemia $(>20$ $\mathrm{mmol} / \mathrm{L}$ ) for at least 2 to 4 weeks [63]. The authors state further, that children with IF but without IFALD frequently have an isolated increase in transaminases or a moderate increase in GGT (usually < $150 \mathrm{IU} / \mathrm{L}$ ) [63]. Therefore, we decided to considered a time period of 14 days pathological.

We found deranged LFTs in 106 patients, 35.0\% of our population. Amongst those, 45/106 (42,5\%, 14.8\% of the total population) experienced an elevation of more than one test. We found the occurence of abnormal LFTs was correlating to the duration of $\mathrm{PN}$ and the longer PN was given the higher the incidence. Our results are in keeping with other reports from the literature. As 21 patients had a concomitant line infection which is a well documented cause of liver inflammation our numbers may have, however been falsely elevated [64].

Early signs of steatosis after two weeks of PN are a mild to moderate elevation of AST and ALT, whilst serum alkaline phosphatase and bilirubin are usually not raised [58]. Cholestasis on the other hand is associated with elevated serum concentrations of ALP, GGT and bilirubin [58]. The majority of our patients (82/303; 27.1\%) had an isolated rise in ALT suggestive of an inflammatory response of the liver or early steatosis. Cholestasis was less common. Steatosis is potentially reversible even if $\mathrm{PN}$ is continued, whilst cholestasis may progress to cirrhosis and liver failure if weaning off PN is not possible [58]. However, a significant number of our patients demonstrated a rise in GGT and bilirubin affecting $38 \quad(12,5 \%)$ and $31 \quad(10,5 \%)$ respectively.
Steatosis can be a consequence of intravenous overnutrition $[47,48]$. Unfortunately we did not have data available on the amount of non nitrogen calories provided per Kcal of body weight and we can hence not comment if too much nutrients could be held responsible.

\section{CVC related complications}

Central venous catheters are integral to pediatric PN administration and their benefits should be carefully balanced against the risk they pose [65-68]. CVC related complication were less common in our population (16.4\%) compared to the NCEPOD audit (25\%) .On the other hand, central line infections were much more common in our cohort of infants $(4 / 67)$ in comparison to the relatively low numbers $(5 / 264)$ in the NCEPOD audit. There is clear need in our very young patients to reduce infection rates.

CLABSIs are a known complication of PN administration [69, 70]; a prolonged catheter dwell time is associated with an increased risk for infection and the microorganisms usually involved are Gram positiva bacteria [71, 72]. CLABSIs account for significant morbidity, mortality and financial burden to the health care system [72].

In our study 31children (10.2\%) developed a central line infection. The mean duration of PN administration was longer in these children than in the group which did not develop any CVC related infection (37 days vs 17 days). Gram positive microorganism were responsible for altmost $71 \%$ of infections, followed by gram negative bacteria $(12.9 \%)$ and candida species $(9.7 \%)$. Our results are simlar to those found in the literature [69-72].

Central venous catheter blockage affected $11.6 \%$ of children. Similar numbers are reported in patients on PN within 1 to 2 years after catheter placement (14\% to $36 \%)$ [73]. Catheter occlusion can negatively impact on patient care: it can lead to a delay or cancellation of medical procedures, potentially interrupt the administration of intravenous medication and nutrition and has a risk of CLABSIs which in turn may lead to catheter removal [74, 75]. We were surprised about the relatively high incidence of early catheter blockage. However, many of the patients requiring chemotherapy for malignancies and those post bone marrow trabsplantation require multiple blood products and their catheter is accessed frequently for a large number of drugs, which may account for the number of catheter related problems encountered.

\section{Nutritional complications: high vitamin $\mathrm{A}$ and $\mathrm{E}$ and low selenium levels}

In children who received $\mathrm{PN}$ longer than 28 days, nutritional complications occured in 35/55 patients. As 18/55 did not have bloods for micronutrients sent the figure may even be higher. The adequate provision of vitamins 
and trace elements intravenously can be challenging and we were hence not surprised by the large number of patients affected. The exact optimal amount of individual vitamins and trace elements children and neonates require remains a matter of debate, and only scanty evidence is available from the literature in the last 20 years [76]. In particular, little is know about the micronutrient requirements in children with acute and chronic disease. theri needs may be significantly different to those of healthy children [6].

The ESPGHAN guidelines state that the actual amount of vitamins delivered to the patient through PN may be much lower than the intended dose as vitamins degradate over time in the PN bag [6]. We were hence suprised to see the number of children who had high levels of vitamin A and E. In our PN compounding unit fat soluble vitamins are added to the lipid emulsion at the following concentration: retinol $230 \mathrm{IU}$ per Kg, maximum dose $2300 \mathrm{IU}$, which is in keeping with the ESPGHAN guidelines of 2005; alfa-tocoferol 0.7 IU per Kg, maximum $70 \mathrm{IU}$, which is well below the recommended dose $(2.8-3.5 \mathrm{IU} / \mathrm{kg})$. Both vitamins tend to leach into the giving set which may lead to further losses [77]. We therefore expected a deficiency of these vitamins, particularly vitamin E. However, we found high levels of vitamin $\mathrm{E}$ and $\mathrm{A}$ in $27.3 \%$ and $25.5 \%$ of children recespectivley who received inpatient $\mathrm{PN}$ for more than one month. Both hypervitaminosis and hypovitaminosis A have been reported in patients who are fully PN dependant [78-80]; Hack et al found elevated vitamin A levels in approximately one fourth of their patients [81]. Although their results are comparable to ours, their patients received much higher doses than ours $(214 \%$ of the recommended dose). Hypovitaminosis $\mathrm{E}$ is more frequently reported in children receiving PN [82]. In contrast, we found a large prevalence of high vitamin $\mathrm{E}$ levels in our group of children. We were concerned our results were falsely elevated tue to the way they were analysed in our lab. However, the method used (liquid chromatography) excludes falsely elevated vitamin $\mathrm{E}$ levels and our results hence appear to be genuine. No child in our study or in the study by Hack et el demonstrated any symptoms associated with vitamin A or E toxicity [81]. Multicenter studies or a national database such as the paediatric electronic British Articificial Nutrition Survey (eBANS) may be useful in identifying if children receiving PN are genuinely at risk of high fat soluble vitamin levels or whether there is a subgroup of patients who is more vulnerable.

When anlysing our trace element results we found low levels of selenium in altmost $30 \%$ of children who received PN for more than 28 days. Selenium deficiency can lead to significant complications including cardiomyopathy, arthritis, skeletal myopathy, loss of pigmentation of hair and skin and macrocytosis [83]. Increased transaminases and creatinine kinase have been noted in patients on long-term parenteral nutrition without selenium supplementation [83]. In children with less than $15 \mathrm{~kg}$ body weight, PN bags compounded by our PN pharmacy provide 25 nanomol $/ \mathrm{kg} /$ day and in those with more than 15 $\mathrm{Kg} 4 \mathrm{nanomol} / \mathrm{kg} /$ day. Both are in keeping with current ESPGHAN guidelines. However, the precise requirements for trace elements in parenteral provision remain still a matter of debate and alterations in the blood levels of children on $\mathrm{PN}$ as in our group may hence be not suprising [76]. The results should be interpreted with caution though, as plasma selenium levels reflect dietary intake rather than selenium stores or bioavailability [84]. Selenium-dependent enzyme activity in tissue and body fluids in addition to serum selenium levels may hence be a better measure $[6,85]$.

Our results suggest thats methods aiming to detect micronutrient stores and bioavailability should be developed to detect a truly deficient state or potentially toxic levels prior to manifestations of clinical symptoms.

\section{Conclusions}

Despite the advancements made since PN was first prescribed to children in the late $1960 \mathrm{~s}$, PN is still prescribed inappropriately and complications occur frequently in the hospital setting. There is clearly still work to be done to improve inpatient prescribing of PN to children. The indications for PN have evolved over the last forty years. Initially $\mathrm{PN}$ was prescribed to patients with a primary pathology of the GI tract [86]. However, in recent years PN is now commonly used for nutritional support in other conditions [16]. This is strongly supported by our data, as the majority of children received $\mathrm{PN}$ for a other conditions than a primary GI pathology (61.7\% non GI vs $38.3 \%$ GI). It is still not clear how the critically ill child should be fed and both optimal route of feeding (enteral versus parenteral) and timing for the start of nutritional support is subject to debate [87]. Given that more and more children are admitted to paediatric intensive care units urgent randomized controlled trials are needed to guide physicians in the decision making process. Moreover consultation of a NST has been shown to reduce the number of children receiving short term PN unnecessarily. When a NST is consulted enteral feeds are more frequently started early and the incidence of catheter related and metabolic complications reduced. Utilisation of an NST therefore reduces the burden of care and is cost effective [88, 89]. Our hospital is staffed with a NST, and children receiving PN are reviewed once a week. However, it is currently not routine practice to consult this team prior to the start of PN, which may lead to overprescribing of PN. Studies on adults have shown that mandatory 
involvement of the NST prior to the start of PN reduced the number of inappropriate PN prescriptions [89]. We will therefore propose to our hospital executive board that the same policy should be put in place in our unit in the future. Moreover, given the high percentage of children developing complications, it might also be of benefit to review these most vulnerable of patients more frequently. Future staffing numbers should take this into consideration.

\begin{abstract}
Abbreviations
ALP: Alkaline phosphataseALTAlanine aminotransferase; AST: Aspartate aminotransferase; CLABSIs: Central line associated bloodstream infections; CVC: Central venous catheter; eBANS: Paediatric electronic British Articifical Nutrition Survey; ESPGHAN: European Society of Paediatric Gastroenterology, Hepatology and Nutrition; GGT: Gamma-glutamyl transpeptidase; HPLC: High pressure liquid cromatography; IF: Intestinal failure; LFTs: Liver function tests; NCEPOD: National Confidential Enquiry into patient outcome and death; NST: Nutrition Support Team; PN: Parenteral Nutrition; RS: Refeeding syndrome; TG: Triglycerids; uNa: Urinary sodium
\end{abstract}

\section{Funding}

No sources of funding to declare.

The first author received recently after writing the article a grant from the Italian Society of Pediatrics (SIP/Dicofarm) to publish this work.

\section{Availability of data and materials}

All data generated or analysed during this study are included in this published article.

\begin{abstract}
Authors' contributions
CM conception and design of the study, acquisition of data, analysis and interpretation of data, main drafting of the manuscript and revising it critically for important intellectual conten. NL conception and design of the study, acquisition of data, analysis and interpretation of data. GZ gave final approval of the version to be published. JK involved in conception and design of the study, drafting of the manuscript and revising it critically for important intellectual content. Agreed to be accountable for all aspects of the work in ensuring that questions related to the accuracy or integrity of any part of the work were appropriately investigated and resolved. Gave final approval of the version to be published.
\end{abstract}

\section{Ethics approval and consent to participate}

The study was approved by the Ethical Committee of Great Ormond Street Hospital.

\section{Consent for publication}

Informed consent was obtained from parents or legal guardians of the patients.

\section{Competing interests}

The authors declare that they have no competing interests.

\section{Publisher's Note}

Springer Nature remains neutral with regard to jurisdictional claims in published maps and institutional affiliations.

Received: 12 January 2018 Accepted: 25 May 2018

Published online: 08 June 2018

\section{References}

1. D'Antiga L, Goulet O. Intestinal failure in children: the European view. J Pediatr Gastroenterol Nutr. 2013;56:118-26.

2. Willmore DE, Dudrick SJ. Growth and develpment of an infant receiving all nutrients exclusively by vein. JAMA. 1968;203:860-4.

3. Jeejeebhoy KN. Total parenteral nutrition: potion or poison? Am J Clin Nutr. 2001;74:160-3.
4. Maurer J, Weinbaum F, Turner J, Brady T, Pistone B, D'Addario V, et al. Reducing the inappropriate use of parenteral nutrition in an acute care teaching hospital. JPEN J Parenter Enteral Nutr. 1996;20:272-4.

5. Braegger C, Decsi T, Dias JA, Hartman C, Kolacek S, Koletko B, et al. (2010) Practical Approach to Paediatric Enteral Nutrition?? A Comment by the ESPGHAN Committee on Nutrition. J Pediatr Gastroenterol Nutr. 2010;51:110-22.

6. Koletzko B, Goulet O, Hunt J, Krohn K, Shamir R. Guidelines on Paediatric Parenteral Nutrition of the European Society of Gastroenterology, Hepatology and Nutrition (ESPGHAN) and the European Society for Clinical Nutrition and Metabolism (ESPEN), Supported by the European Society of Paediatric Research (ESPR). J Pediatr Gastroenterol Nutr. 2005;41(2):S1-4.

7. Agudelo GM, Giraldo NA, Aguilar NL, Restrepo BE, Vanegas M, Alzate S, et al. Incidence of nutritional support complications in patient hospitalized in wards. multicentric study. Colomb Med (Cali). 2012;43:147-53.

8. Herranz Antolín S, Álvarez De Frutos V, Blasco Guerrero M, García Martínez Mdel C, Gimeno Fernández Mdel C. Nutritional support with parenteral nutrition Course and associated complications. Endocrinol Nutr. 2013;60: 287-93.

9. Stewart J A D, Mason D G, Smith N, Protopapa K, Mason M. A Mixed Bag: an enquiry into the care of hospital patients receiving parenteral nutrition. $A$ report by the National Confidential Enquiry into Patient Outcome and Death 2010. http://www.ncepod.org.uk/2010report1/downloads/PN_report.pdf.

10. Physical status: the use and interpretation of anthropometry. Report of a WHO Expert Committee. World Health Organ Tech Rep Ser. 1995;854:1-452.

11. Embleton ND, Simmer K. Practice of Parenteral Nutrition in VLBW and ELBW Infants. In: Koletzko B, Poindexter B, Uauy R, editors. Nutritional care of Preterm infants: Scientific Basis and Practical Guidelines World Review of Nutrition and Dietetics, vol. 110; 2014. p. 177-89.

12. Rhoda KM, Porter MJ, Quintini C. Fluid and Electrolyte Management: Putting a Plan in Motion. JPEN J Parenter Enteral Nutr. 2011;35:675-85.

13. Shulman RJ, Sarah P. Parenteral Nutrition in Infants and Children. J Pediatric Gastroenteroly and Nutrition. 2004;36:587-607.

14. Arsenault D, Brenn M, Kim S, Gura K, Compher C, Simpser E, A.S.P.E.N, et al. Clinical Guidelines: Hyperglycemia and Hypoglycemia in the Neonate Receiving Parenteral Nutrition. JPEN J Parenter Enteral Nutr. 2012;36:81-95.

15. Fanelli C, Pampanelli S, Epifano L, Rambotti AM, Ciofetta M, Modarelli F, et al. Relative roles of insulin and hypoglycaemia on induction of neuroendocrine responses to, symptoms of, and deterioration of cognitive function in hypoglycaemia in male and female humans. Diabetologia. 1994; 37:797-807.

16. ASPEN Board of Directors. Guidelines for the use of parenteral and enteral nutrition in adult and pediatric patients. American Society for Parenteral and Enteral Nutrition. JPEN J Parenter Enteral Nutr. 2013;17:1SA-52SA.

17. Duranton F, Depner TA. MD, and Argilés A, MD. The Saga of Two Centuries of Urea: Nontoxic Toxin or Vice Versa? Semin Nephrol. 2014;34:87-96.

18. Rockey DC. Gastrointestinal bleeding. In: Feldman M, Friedman LS, Sleisenger MH, editors. Sleisenger \& Fordtran's Gastrointestinal and Liver Disease. 7th ed. Philadelphia: Saunders; 2002. p. 211-48.

19. Centers for Disease Control and Prevention. Guidelines on central line associated bloodstream infections (CLABSI) event. https://www.cdc.gov/ nhsn/pdfs/pscmanual/4psc_clabscurrent.pdf.

20. Fathmy M, Young SP. Modulation of iron metablism in monocyte cell line U937 by inflammaatory cytokines: changes in transferin uptake, iron handling and ferritin mRNA. Bichem J. 1993;296:175-81.

21. Brion L, Bell E, Raghuveer T. Vitamin E supplementation for prevention of morbidity and mortality in preterm infants. Cochrane Database Syst Rev. 2003:4:CD003665

22. Greene HL, Hambidge M, Schanler R, Tsang R. Guidelines for the use of vitamins, trace elements, calcium, magnesium, and phosphorus in infant and children receiving total parenteral nutrition: report of the Subcommittee on Pediatric Parenteral Nutrient Requirements from the Committee on Clinical Practice Issues of the American Society for Clinical Nutrition. Am J Clin Nutr. 1988;48:1324-42.

23. O'Brien DD, Hodges RE, Day AT, et al. Recommendations of nutrition support team promote cost containment. JPEN J Parenter Enteral Nutr. 1986;10:300-2.

24. Hendricks KM, Duggar C, Gallagher L, et al. Malnutrition in hospitalized paediatric patients. Current prevalence Arch Pediatr Adolesc Med. 1995;149: 1118-22.

25. Mehta NM, Compher C, A.S.P.E.N, et al. Clinical guidelines: nutrition support of the critically ill child. JPEN J Parenter Enteral Nutr. 2009;33:260. 
26. Braga $M$, Ljungvuist $O$, Soeters $P$, et al. ESPEN guidelines on parenteral nutrition: surgery. Clin Nutr. 2009;28:378.

27. Tentori F, Blayney MJ, Albert JM, et al. Mortality risk for dialysis patients with different levels of serum calcium, phosphorus, and PTH: the Dialysis Outcomes and Practice Patterns Study (DOPPS). Am J Kidney Dis. 2008;52: 519-30.

28. Hästbacka J, Pettila V. Prevalence and predictive value of ionized hypocalcemia among critically ill patients. Acta Anaesthesiol Scand. 2003;47:1264-9.

29. Liamis G, Rodenburg EM, Hofman A, et al. Electrolyte disorders in community subjects: prevalence and risk factors. Am J Med. 2013;126: 256-63.

30. Qunibi WY. Consequences of hyperphosphatemia in patients with endstage renal disease (ESRD). Kidney Int Suppl. 2004;90:S8-S12.

31. Schucker JJ, Ward KE. Hyperphosphatemia and phosphate binders. Am J Health-Syst Pharm. 2005;62:2355-61.

32. Janigan D, Hirsch D, Klassen $G$, et al. Calcified subcutaneous arterioles with infarcts of the subcutis and skin ("calciphylaxis") in chronic renal failure. Am J Kidney Dis. 2000;35:588-97.

33. Rude RK. Magnesium depletion and hypermagnesemia. In: Rosen CJ, Compston JE, Lian JB, editors. Primer on the metabolic bone diseases and dis- orders of mineral metabolism. Hoboken (NJ): Wiley; 2009. p. 325-8.

34. Amita T, Timothy C, Simon B. Hypercalcemia in acute lymphoblastic leukemia: an overview. J Pediatr Hematol Oncol. 2009:31:424-7.

35. Muna Q, Ibrahim A, Rudolph PV, et al. Hypercalcemia in pediatric acute megakaryocytic leukemia. J Pediatr Hematol Oncol. 2009;31(5):373-6.

36. Shiber JR, Mattu A. Serum phosphate abnormalities in the emergency depart- ment. J Emerg Med. 2002;23:395-400.

37. Geerse DA, Bindels AJ, Kuiper MA, et al. Treatment of hypophosphatemia in the intensive care unit: a review. Crit Care. 2010;14:R147.

38. Crook MA, Hally V, Panteli JV. The importance of refeeding syndrome Nutrition. 2001;17:632-7.

39. Rio A, Whelan K, Goff L, Reidlinger DP, Smeeton N. Occurrence of refeeding syndrome in adults started on artificial nutrition support: prospective cohort study. BMJ Open. 2013;11:3.

40. Hortencio TD, Nogueira RJ, de Lima Marson FA, Ribeiro AF. Hypophosphatemia, Hypomagnesemia, and Hypokalemia in Pediatric Patients Before and During Exclusive Individualized Parenteral Nutrition. Nutr Clin Pract. 2016;31:223-8.

41. Gore DC, Chinkes D, Heggers J, Herndon DN, Wolf SE, Desai M. Association of hyperglycemia with increased mortality after severe burn injury. J Trauma. 2001;51:540-4

42. Umpierrez GE, Isaacs SD, Bazargan N, et al. Hyperglycemia: an independent marker of in-hospital mortality in patients with undiagnosed diabetes. J Clin Endocrinol Metab. 2002;87:978-82.

43. Sleiman I, Morandi A, Sabatini T, et al. Hyperglycemia as a predictor of inhospital mortality in elderly patients without diabetes mellitus admitted to a sub-intensive care unit. J Am Geriatr Soc. 2008;56:1106-10.

44. McCowen KC, Bistrian BR. Hyperglycemia and nutrition support: Theory and Practice. Nutr Clin Pract. 2004;9:235-44.

45. Clement S, Braithwaite SS, Ahmann A, et al. Management of Diabetes and Hyperglycemia in Hospitals. Diabetes Care. 2004:27:553-91.

46. Olveira G, Tapia MJ, Ocón J, et al. Study Group of Hyperglycemia in Parenteral Nutrition: Nutrition Area of the Spanish Society of Endocrinology and Nutrition (SEEN): Parenteral nutrition-associated hyperglycemia in noncritically ill inpatients increases the risk of in-hospital mortality (multicenter study). Diabetes Care. 2013;36:1061-6.

47. Robin AP, Carpentier YA, Askanazi J, Nordenström J, Kinney JM. Metabolic consequences of hypercaloric glucose infusions. Acta Chir Belg. 1981;80: $133-40$.

48. Elwyn DH, Askanazi J, Kinney JM, Gump FE. Kinetics of energy substrates. Acta Chir Scand Suppl. 1981:507:209-19.

49. Nose O, Tipton JR, Ament ME, Yabuuchi $\mathrm{H}$. Effect of the energy source on changes in energy expenditure, respiratory quotient, and nitrogen balance during total parenteral nutrition in children. Pediatr Res. 1987;21:538-41.

50. Burke JF, Wolfe RR, Mullany CJ, Mathews DE, Bier DM. Glucose requirements following burn injury. Parameters of optimal glucose infusion and possible hepatic and respiratory abnormalities following excessive glucose intake. Ann Surg. 1979;190:274-85.

51. Tulikoura I, Huikuri K. Morphological fatty changes and function of the liver, serum free fatty acids, and triglycerides during parenteral nutrition. Scand J Gastroenterol. 1982;17:177-85.
52. Hays S, O'Brian Smith E, Sunehag A. Hyperglycemia is a risk fac- tor for early death and morbidity in extremely low birth-weight infants. Pediatrics. 2006; 118:1811-8.

53. Alaedeen D. Total parenteral nutrition associated hyperglycemia correlates with prolonged mechanical ventilation and hospital stay in septic infants. J Pediatr Surg. 2006;41:239-44.

54. Sunehag $\mathrm{A}$. The role of parenteral lipids in supporting gluconeogenesis in the very premature infants. Pediatr Res. 2003;54:480-6.

55. Murdock N, Crighton A, Nelson L, Forsyth J. Low birthweight infants and total parenteral nutrition immediately after birth. II. Randomized study of biochemical tolerance to intravenous glucose, amino acids and lipid. Arch Dis Child. 1995;73:F8-F12.

56. Arsenault D, Brenn M, Kim S, Gura K, Compher C, Simpser E, A.S.P.E.N, et al. Clinical Guidelines: hyperglycemia and hypoglycemia in the neonate receiving parenteral nutrition. JPEN J Parenter Enteral Nutr. 2012;36:81-95.

57. Lauriti G, Zani A, Aufieri R, Cananzi M, Chiesa PL, Eaton S, et al. Incidence, Prevention, and Treatment of Parenteral Nutrition Associated Cholestasis and Intestinal Failure Associated Liver Disease in Infants and Children: A Systematic Review. JPEN J Parenter Enteral Nutr. 2014;38:70-85.

58. Kumpf VJ. Parenteral Nutrition-Associated Liver Disease in Adult and Pediatric Patients. Nutr Clin Pract. 2006;21:279-90.

59. Quigley EMM, Marsh MN, Shaffer JL, Markin RS. Hepatobiliary complications of total parenteral nutrition. Gastroenterology. 1993;104:286-301.

60. Kelly DA. Liver complications of pediatric parenteral nutrition: epidemiology. Nutrition. 1998;14:153-7.

61. Beath SV, Davies P, Papadopoulou A, et al. Parenteral nutritionrelated cholestasis in postsurgical neonates: multivariate analysis of risk factors. J Pediatr Surg. 1996;31:604-6.

62. Teitelbaum DH. Parenteral nutrition-associated cholestasis. Semin Pediatr Surg. 2001;10:72-80

63. Lacaille F, Gupte G, Colomb V, D'Antiga L, Hartman C, Hojsak I, et al. Intestinal Failure-Associated Liver Disease: A Position Paper of the ESPGHAN Working Group of Intestinal Failure and Intestinal Transplantation. J Pediatr Gastroenterol Nutr. 2015:60:272-83.

64. Wolf A, Pohlandt F. Bacterial infection: the main cause of acute cholestasis in newborn infants receiving short-term parenteral nutrition. J Pediatr Gastroenterol Nutr. 1989;8:297-303.

65. Simon A, Fleischhack G, Hasan C, Bode U, Engelhart S, Kramer MH. Surveillance for nosocomial and central line-related infections among pediatric hematologyoncology patients. Infect Control Hosp Epidemiol. 2000;21:592-6.

66. Dudeck MA, Horan TC, Peterson KD, Allen-Bridson K, Morrell G, Pollock DA, et al. National Healthcare Safety Network (NHSN) Report, data summary for 2010, device-associated module. Am J Infect Control. 2011;39:798-816.

67. Kelly M, Conway M, Wirth K, Potter-Bynoe G, Billett AL, Sandora TJ. Moving CLABSI prevention beyond the intensive care unit: Risk factors in pediatric oncology patients. Infect Control Hosp Epidemiol. 2011;32:1079-85.

68. Dannenberg C, Bierbach U, Rothe A, Körholz D. Ethanol-lock technique in the treatment of bloodstream infections in pediatric oncology patients with broviac catheter. J Pediatr Hematol Oncol. 2003;25:616-21.

69. Blot SI, Depuydt P, Annemans L, Benoit D, Hoste E, De Waele JJ, et al. Clinical and economic outcomes in critically ill patients with nosocomial catheter-related bloodstream infections. Clin Infect Dis. 2005;41:1591-8.

70. Saint S, Veenstra DL, Lipsky BA. The clinical and economic con- sequences of nosocomial central venous catheter-related infection: are antimicrobial catheters useful? Infect Control Hosp Epidemiol. 2000;21:375-80.

71. Sengupta A, Lehmann C, Diener-West M, Perl TM, Milstone AM. Catheter duration and risk of CLA-BSI in neonates with PICCs. Pediatrics. 2010;125:648-53.

72. Smith RN, Nolan JP. Central venous catheters. BMJ. 2013;347:f6570.

73. Ernst FR, PharmD EC, Lipkin C, Tayama D, Amin AN. Comparison of Hospital Length of Stay, Costs, and Readmissions of Alteplase Versus Catheter Replacement Among Patients With Occluded Central Venous Catheters. J Hosp Med. 2010:9:490-6.

74. Hadaway LC. Reopen the pipeline. Nursing. 2005;35:54-61.

75. Mayo DJ, Pearson DC. Chemotherapy extravasation: a consequence of fibrin sheath formation around venous access devices. Oncol Nurs Forum. 1995; 22:675-80.

76. Shulman RJ. New developments in total parenteral nutrition for children. Curr Gastroenterol Rep. 2000;2:253-8.

77. Haas C, Genzel-Boroviczeny O, Koletzko B. Losses of vitamin A and E in parenteral nutrition suitable for premature infants. Eur J Clin Nutr. 2002;56: 906-12. 
78. Greene HL, Hambidge KM, Schanler R, Tsang RC. Guidelines for the use of vitamins, trace elements, calcium, magnesium, and phosphorus in infants and children receiving total parenteral nutrition: report of the Subcommittee on Pediatric Parenteral Nutrient Requirements from the Committee on Clinical Practice Issues of the American Society for Clin Nutr. Am J Clin Nutr. 1988:48:1324-42.

79. Howard L, Chu R, Feman S, Mintz H, Ovesen L, Wolf B. Vitamin A deficiency from long-term parenteral nutrition. Ann Intern Med. 1980;93:576-7.

80. Seibert JJ, Byrne WJ, Golladay ES. Development of hypervitaminosis A in a patient on long-term parenteral hyperalimentation. Pediatr Radiol. 1981;10: 173-4.

81. Hack SL, Merritt RJ, Morgan RM, Keefe MT. Serum vitamin A and E concentrations in pediatric total parenteral nutrition patients. JPEN J Parenter Enteral Nutr. 1990;14:189-94.

82. Thurlow PM, Grant JP. Vitamin E and total parenteral nutrition. Ann N Y Acad Sci. 1982;393:121-32.

83. Wong T. Parenteral trace elements in children: clinical aspects and dosage recommendations. Curr Opin Clin Nutr Metab Care. 2012;15:649-56.

84. Ashton K, Hooper L, Harvey LJ, Hurst R, Casgrain A, Fairweather-Tait SJ. Methods of assessment of selenium status in humans: a systematic review. Am J Clin Nutr. 2009;89:2025S-39S.

85. Loui A, Raab A, Braetter $P$, et al. Selenium status in term and preterm infants during the first months of life. Eur J Clin Nutr. 2007;62:349-55.

86. Dudrick SJ, Wilmore DW, Vars HM, et al. Long-term total parenteral nutrition with growth, development, and positive nitrogen balance. Surgery. 1968;64: 134-42.

87. Joffe A, Anton N, Lequier L, Vandermeer B, Tjosvold L, Larsen B, Hartling L. Nutritional support for critically ill children. Cochrane Database Syst Rev. 2009;15:CD005144

88. Kennedy JF, Nightingale JM. Cost savings of an adult hospital nutrition support team. Nutrition. 2005;21:1127-33.

89. Roberts MF, Levine GM. Nutrition support team recommendations can reduce hospital costs. Nutr Clin Pract. 1992;7:227-30.

90. ASPEN Board of Directors and the Clinical Guidelines Task Force. Guidelines for the use of parenteral and enteral nutrition in adult and pediatric patients. JPEN J Parenter Enteral Nutr. 2002;26:1SA-138SA.

\section{Ready to submit your research? Choose BMC and benefit from:}

- fast, convenient online submission

- thorough peer review by experienced researchers in your field

- rapid publication on acceptance

- support for research data, including large and complex data types

- gold Open Access which fosters wider collaboration and increased citations

- maximum visibility for your research: over $100 \mathrm{M}$ website views per year 